\title{
Bridging the Data Gap in Breast Cancer Risk Assessment to Enable Widespread Clinical Implementation across the Multiethnic Landscape of the US
}

\author{
Erika Spaeth', Athena Starlard-Davenport' ${ }^{2}$, Richard Allman ${ }^{3}$ \\ 'Phenogen Sciences Inc, Charlotte, NC 28269, USA \\ ${ }^{2}$ Department of Genetics, Genomics and Informatics, University of Tennessee Health Science Center, Memphis, TN 38163, USA \\ ${ }^{3}$ Genetic Technologies, Ltd, Fitzroy, VIC 3065, Australia
}

Article Info

\section{Article Notes}

Received: June 01, 2018

Accepted: August 03, 2018

\section{${ }^{*}$ Correspondence:}

Dr. Erika Spaeth, Phenogen Sciences, Charlotte, NC 28269,

USA; Telephone No: +1.704-926-5700;

Email: Erika.spaeth.tuff@phgns.com

() 2018 Spaeth $E$. This article is distributed under the terms of the Creative Commons Attribution 4.0 International License.

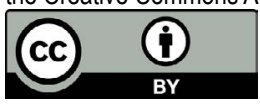

Keywords:

Breast cancer risk assessment

Prevention; Screening

General population

Ethnicity

Race

Polygenic risk

\section{ABSTRACT}

Breast cancer remains the second leading cause of cancer death among women and is the most commonly diagnosed cancer in women. Breast cancer risk assessment has been clinically available for nearly 30 years yet is under-utilized in practice for multiple reasons. Incorporation of polygenic risk as well as breast density measurements, promise to increase the accuracy of risk assessment. With that comes the hope that both prevention and screening become more personalized and thus more effective. Incidence rates have been static over the past 15 years and have even increased slightly in African American and Asian/Pacific Islander populations despite the robust data on breast cancer risk reduction measures that exist. Current challenges in reducing breast cancer incidence begin with robust data curation that allows for appropriate risk stratification across our multiethnic population and conclude with the implementation of prevention strategies within our fractured healthcare system.

\section{Introduction}

Breast cancer risk assessment research has been ongoing for decades. Large population studies have enabled scientists and clinicians to identify risk factors that influence a woman's risk of being diagnosed with breast cancer. After all, one in eight women in the US is diagnosed with breast cancer in their lifetime. Yet, with all the accumulated evidence of breast cancer risk, we still face several obstacles in implementing appropriate preventative and screening measures for high-risk women for two main reasons: first, because many women aren't being assessed for their risk and second because women aren't being offered or accepting of preventative therapy options that have been shown to reduce the risk of developing breast cancer. Moreover, these measures continue to lag behind for racial and ethnic minorities in the US whether for socioeconomic reasons associated with the limited clinical interaction, discrimination in care or because to date, most large studies have an overwhelming majority of non-Hispanic white subjects. This short review attempts to summarize the polarizing evidence supporting preventative therapy in high-risk women and our inability as a medical community to implement general population risk assessment strategies for the identification of high-risk women that would benefit from this therapy. 


\section{Chemoprevention Trials Support the Clinical Utility of Breast Cancer Risk Assessment Models}

Cost-effectiveness drives general population screening measures; this is one of the many principles of population screening adopted by the World Health Organization in $1968^{1}$. One of the earliest studies that were designed to demonstrate the feasibility of breast cancer screening was the Breast Cancer Detection Demonstration Project that began in the 1970s and initially enrolled $\sim 280,000$ women, and a longer-term follow up study of $\sim 55,000$ women $^{2,3}$. Not only did this study show that mammography was able to detect non-palpable invasive and non-invasive breast cancers, it showed a survival advantage for screened women. These data provided the foundation upon which the Gail model was to be developed ${ }^{4}$. The Gail model was the gold standard used in the subsequent trial design to establish the criteria for a "high-risk" woman (5-year risk $\geq 1.67 \%$ ) in the National Surgical Adjuvant Breast and Bowel Project, Prevention-1 (NSABP P-1) study, also called the Breast Cancer Prevention Trial (BCPT). This trial supported the efficacy of Tamoxifen as a risk-reducing medication for breast cancer prevention by showing a nearly $50 \%$ reduction in invasive breast cancers compared to the placebo $\mathrm{arm}^{5}$. A subsequent trial (NSABP2/STAR) supported the efficacy of another selective estrogen receptor modulator (SERM), Raloxifene in breast cancer risk reduction ${ }^{6}$. By 2007, both SERM were FDA approved for use in breast cancer prevention. Building off of the momentum from the SERM prevention trials but looking to improve upon observed side-effects, the use of aromatase inhibitors, anastrozole, exemestane or letrozole in postmenopausal women has undergone investigation over the last 15 years $^{7}$. Improved efficacy of aromatase inhibitors in breast cancer prevention trials along with the decrease in toxicity compared to SERMs is promising ${ }^{8,9}$. The United Kingdom's National Institute of Health and Care Excellence (NICE) currently recommends offering anastrozole to the postmenopausal woman at high-risk of breast cancer as do the United States' National Comprehensive Cancer Network (NCCN) and the American Society of Clinical Oncologists (ASCO). Despite the solid evidence offered in the above studies, the rates of breast cancer incidence are no longer decreasing, in fact, they are predicted to continue to increase due to population increases ${ }^{10}$. It is both fiscally and medically responsible to actively attempt chemoprevention measures in high-risk women in order to suppress the impact of breast cancer incidence within our ageing population.

\section{Improving on Breast Cancer Risk Assessment Models}

Insight gained over 30 years of clinical trial data supporting the use of chemoprevention in high-risk women includes the notion that breast cancer risk assessment can appropriately identify high-risk women. Now the task remains to improve upon the risk assessment models currently in use. Recently, the addition of mammographic density and low penetrance alleles associated with breast cancer incidence have improved the discrimination of current risk assessment models ${ }^{11-15}$ Polygenic risk can be broadly described as a value that combines many low penetrant alleles, identified from genome wide association studies (GWAS), and show some predictive ability across a range of complex traits and diseases ${ }^{16-20}$. It should be emphasized that the majority of these identified alleles remain to have an associative rather than causal relationship to breast cancer and direct mechanism of action remain to be elucidated due to the multifactorial nature of the disease. Comparable to a single dot of paint in a neo-impressionism pointillism work of art, the low penetrant nature of any single allele is largely uninformative on its own, but multiple alleles in combination reveal "a larger picture" of a woman's breast cancer risk. Polygenic risk has been largely independent of any other risk factor currently included in risk assessment algorithms with a small attenuation of family history ${ }^{21}$. Previous reports have shown that most SNPs and environmental or clinical risk factors combine multiplicatively, enabling the integration of a polygenic risk score and environmental/clinical factors to be incorporated into risk models ${ }^{22}$.

Even with the improvement of current risk assessment models, these same models for ethnic and racial minorities in the US continue to lag behind. Particularly when it comes to polygenic risk, studies support an impressive improvement in discriminatory accuracy, however, this does not translate across all ethnicities because of the background genetic differences between populations. This is especially important as women of African ancestry are disproportionately affected by higher rates of aggressive forms of breast cancer that are nonresponsive to hormonal therapies compared to other ethnicities ${ }^{23,24}$. Allman et al made the first attempt to incorporate known markers of polygenic breast cancer risk into assessment models for Black and Hispanic populations in the US ${ }^{13}$. We must continue to build on this by identifying additional low penetrance markers associated with women based on their genetic ancestry in order to provide the most accurate risk assessment model. Studies continue to identify additional discriminatory markers that are distinct within one genetic background versus another ${ }^{25-27}$, but larger data sets are needed to provide a robust analysis.

\section{Implementation of Screening in High-Risk Women}

It is important to note that the routine implementation of screening mammogram in the general population has led to a significant reduction in mortality, which may continue to improve as screening technologies evolve. Metaanalyses of general population breast cancer screening mammography in observational case/control studies 
found a significant reduction in breast cancer mortality of $48 \%{ }^{28}$. Despite these data, there is still confusion amongst the inconsistencies of current guidelines due to the weight of benefits versus harms of screening. While a benefit/harm ratio is valid, it may be easier to assess and suggest appropriate screening measures for a patient based on a more personalized approach. The NCCN has specific screening guidelines for women at high-risk of breast cancer based on a series of risk factors including family history, risk assessment scores, ionizing radiation history, and lobular carcinoma in situ (LCIS)/atypical hyperplasia (AH) history ${ }^{29}$. However, many women aren't being assessed for their risk of breast cancer so we are not actually identifying the population that should be receiving the option for alternative screening regimens. The most simplistic screening mechanism may be to assess polygenic risk. Polygenic risk can stratify a woman's risk without any other risk factor, thereby not infringing upon the limited physician-patient interaction during an annual wellness visit. (Figure 1) There are currently ongoing clinical trials attempting to better identify women based on risk/benefit ratio in order to increase compliance with screening,

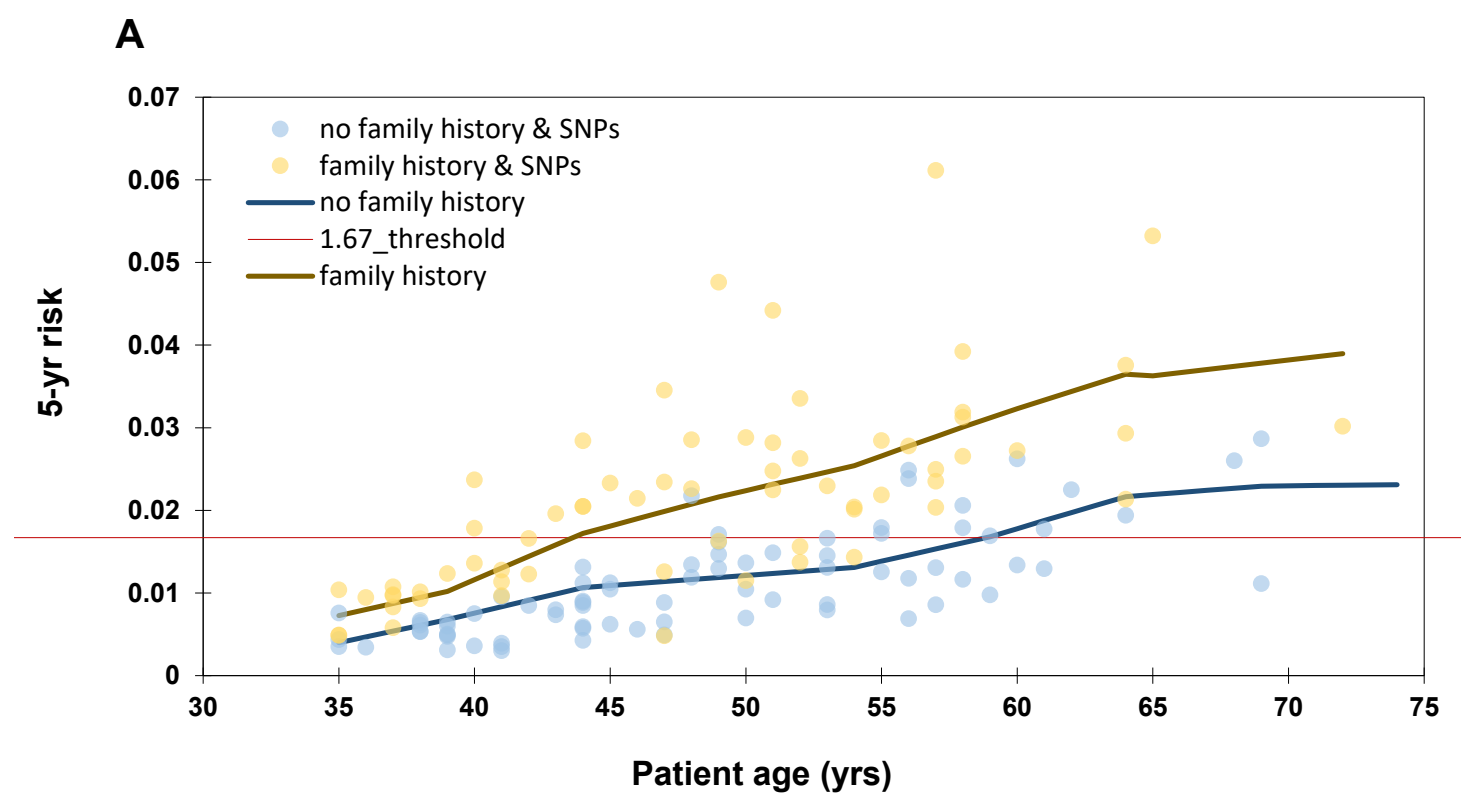

B

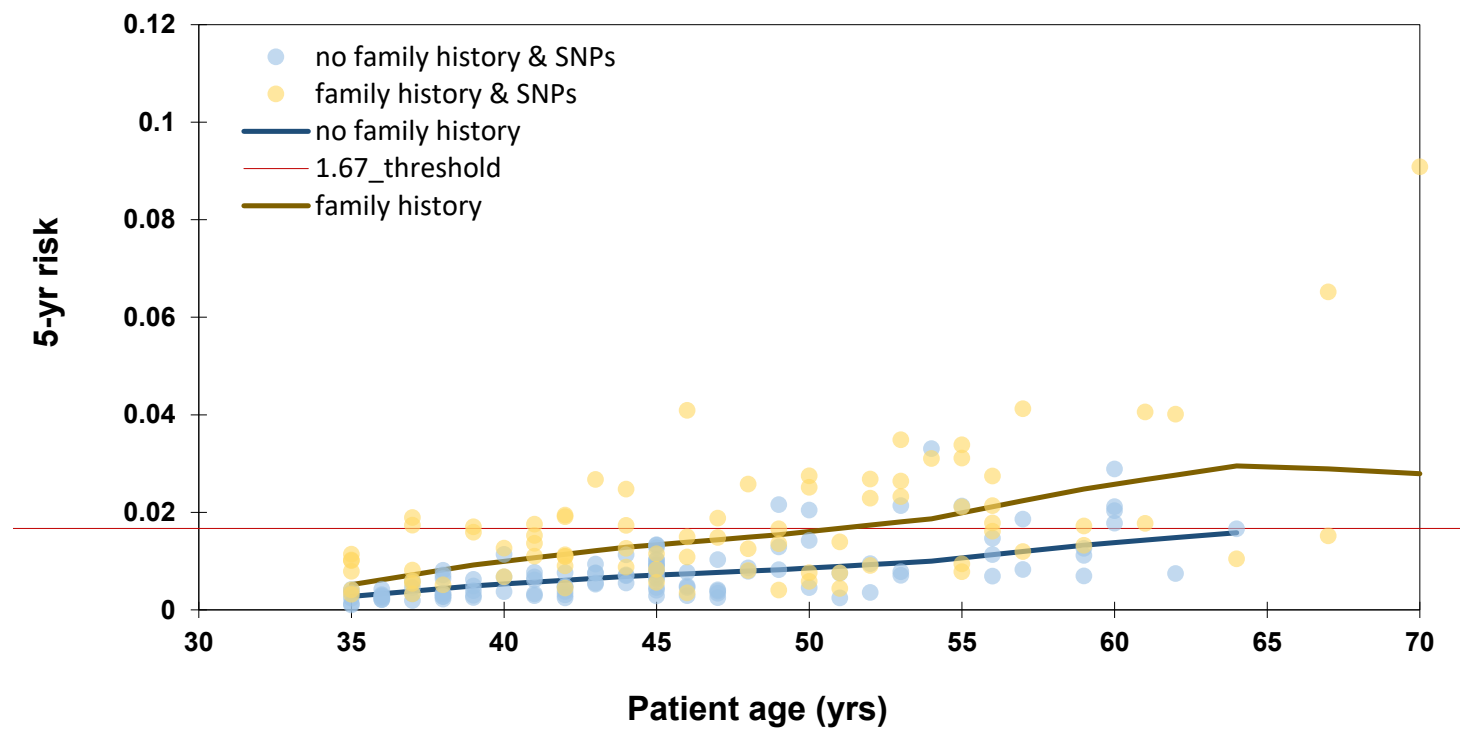

Figure 1. The basic stratification of African American and Hispanic American women based on polygenic risk, after accounting for age and ethnicity. This risk assessment strategy may be an efficient way to screen women for breast cancer risk without encroaching on the overall content covered during the annual wellness visit. (A) African American $(n=144)$ and (B) Hispanic American ( $n=69)$ 5year risk scores stratified by polygenic risk after considering family history (defined as a $1^{\text {st }}$ degree relative with breast cancer) and age. The red line represents the ASCO and NCCN defined 5year high risk threshold of 1.67\%. All patient samples were run in a CLIA lab between 2013 and 2016. 
but also to appropriately identify women who should be screened in a high-risk versus average risk setting ${ }^{30}$.

\section{Implementation of Chemoprevention in High-Risk Women}

The oncology community acknowledges the longstanding implementation hurdles of breast cancer chemoprevention despite numerous clinical trials and thorough objective assessment by National organizations including ASCO, NCCN, ACOG, and USPSTF. These organizations recommend the use of chemoprevention in women whose benefit outweighs the $\mathrm{risk}^{31,32}$. There is a gap in knowledge between the oncology community and the primary care community where the first steps in prevention are taken. Reimers et al show that medical oncology referral and breast cancer risk category to be the strongest predictors of anti-estrogen use $\mathrm{e}^{33}$. A recent study interviewed over 200 physicians regarding their reluctance to prescribe (SERM) chemoprevention. Two of the top five reasons for physician reluctance in prescribing SERM included inefficiency of risk assessment models in clinical practice and the lack of clarity on prevention guidance for physicians ${ }^{34}$. Likewise, studies examining patient reluctance to take chemoprevention medications have been conducted, and one of the central themes, among many, is the trust and opinion of the healthcare provider ${ }^{35}$. Bottom line, the implementation of chemoprevention in high-risk women is dependent on optimal risk assessment models which would help to instill a greater confidence among physicians who play a significant role in the patient's informed decision-making process.

\section{Gaining the Trust of the Medical Community Translates to Patient Trust}

In a recent review by Evans et al, they bring attention to the difficulties in chemoprevention uptake within the breast cancer prevention community but acknowledge that perhaps improved efficacy and reduced "toxicities" of aromatase inhibitors may be a necessary push for appropriate breast cancer chemoprevention ${ }^{36}$. Furthermore, a study showed that patients had limited awareness of preventative options and a preference toward transdermal gel versus a pill ${ }^{37}$. Perhaps a change in chemoprevention approach is necessary to make an impact on breast cancer risk reduction.

Ultimately, if we are not able to provide our entire medical community with the most up to date medical studies and guidance pertaining to breast cancer risk and prevention, we will not see a change in risk assessment or chemoprevention practices. In turn, at-risk women will not be receiving warranted medical options. Estimates of women that could benefit from chemoprevention based on data from the 2000 National Health Interview Survey that takes into account risk/benefit weight of over 2 million eligible high-risk women, amount to nearly 30,000 breast cancers that would be prevented or deferred if those women took chemoprevention over the next 5 years ${ }^{38}$. Moving forward, improving informed discussions between the patient and her physician will only improve comprehensive breast health care for women in the US.

Furthermore, an underlying level of distrust in the medical community still exists across much of the minority populations in the US. There is significant history on which this distrust has been built over the years, a major example being the unethical Tuskegee Syphilis Study where rural African American men in Alabama were maliciously subjected to syphilis to understand the disease and to determine the proper treatment dosage for select populations ${ }^{39}$. More recently, the story of Mrs. Henrietta Lacks, an African American woman whose cervical cancer cells were taken and were commercially developed into the first immortalized cell line (HeLa cells) without her or her family's knowledge has raised concerns about privacy and patients' rights, especially within the African American community ${ }^{40}$. While socioeconomic status and education level also play a role in distrust and continue to impede minority clinical trial recruitment efforts, there is a greater underlying level of distrust that the medical community needs to continue to repair among ethnic minority populations in the US in order to continue to make scientific and medical advancements applicable to our entire population ${ }^{41-43}$. Specifically, interventions focused on alleviating disparities that consider health system change strategies (i.e., changes in health care delivery patterns, health policy, or environmental and community supports that improve health outcomes and health care) are critical for reducing disparities in breast cancer outcomes ${ }^{44}$.

\section{Refine Breast Cancer Risk Assessment into a Better Predictive Tool}

There are ongoing prospective clinical trials looking at the integration of SNP risk into risk assessment for managing chemoprevention including the GENRE (Genetic Risk Estimation of Breast Cancer Prior to Preventive Medication Uptake) Trial out of the Mayo Clinic. The trial provides supporting data from the IBIS-I 20 year follow up showing a $29 \%$ reduction in breast cancer incidence in the tamoxifen arm compared to placebo. However, there was no reduction in breast cancer mortality but an increase in ER negative cases was observed. These findings suggest there may be a subpopulation of women that could benefit from chemoprevention ${ }^{45}$. Could this subpopulation be identified using polygenic risk? A few studies have suggested this hypothesis ${ }^{46,47}$, however further studies are needed to assess this potential benefit.

\section{Conclusion}

Multilayered challenges exist in bringing comprehensive 
breast cancer prevention to women in the US. The scientific community acknowledges that better data sets are needed to incorporate the newest personalized risk stratification approaches to breast cancer assessment across all racial and ethnic backgrounds. There are significant hurdles in acquiring robust ethnic datasets ${ }^{48}$. Particularly in the breast cancer community, significant efforts have been made in acquiring patient cohorts of African-American women such as the AMBER Consortium and the National Cancer Institute's Breast Cancer Genetic Study in AfricanAncestry Populations. Access to such ethnic cohorts enabled the validation of polygenic breast cancer risk assessment in African American and Hispanic populations (in this case using the Women's Health Initiative (WHI) SNP Health Association Resource) ${ }^{13}$. There have also been improvements on Hispanic American risk assessment models ${ }^{51}$ and more recent clinical trials have been successful in accruing more diversified study populations, which is encouraging for the distribution of breast cancer prevention efforts across races/ethnicities ${ }^{33}$.

However, we compound this challenge facing the breast cancer research community because, despite the theoretical focus on prevention, the medical community has not successfully implemented breast cancer prevention strategies based on 30 years of compelling clinical data and multiple existing guidelines. If the research community does develop a risk assessment tool that outperforms current tools, will that be enough to stimulate a change in medical culture? The definition of prevention is the act or practice of keeping something from happening-therefore despite the advocacy from the medical oncology community, the burden of breast cancer prevention is bundled into the ever-expanding preventative care category that PCP and OB/Gyn are expected to carry out during the 15-minute well-woman visit.

\section{Conflict of Interest}

Erika Spaeth is an employee of Phenogen Sciences Inc. and Richard Allman is an employee of Genetic Technologies Ltd.

\section{References}

1. Wilson JJ G. Principles and practices of screening for disease. Geneva, Switzerland: World Health Organization. 1968; Report No.: Public Health Papers No. 34.

2. Morrison AS, Brisson J, Khalid N. Breast cancer incidence and mortality in the breast cancer detection demonstration project [published errtum appears in J Natl Cancer Inst. 1989 Oct 4;81(19):1513. J Natl Cancer Inst. 1988; 80(19): 1540-1547.

3. Baker LH. Breast Cancer Detection Demonstration Project: five-year summary report. CA Cancer. J Clin. 1982; 32(4): 194-225.

4. Gail MH, Brinton LA, Byar DP, et al. Projecting individualized probabilities of developing breast cancer for white females who are being examined annually. J Natl. Cancer Inst. 1989; 81(24): 18791886.
5. Fisher B , Costantino JP, Wickerham DL, et al. Tamoxifen for the prevention of breast cancer: current status of the National Surgical Adjuvant Breast and Bowel Project P-1 study. J Natl Cancer Inst. 2005; 97(22): 1652-1662.

6. Vogel VG. The NSABP Study of Tamoxifen and Raloxifene (STAR) trial. Expert review of anticancer therapy. 2009; 9(1): 51-60.

7. Dunn BK, Ryan A. Phase 3 trials of aromatase inhibitors for breast cancer prevention: following in the path of the selective estrogen receptor modulators. Ann N Y Acad Sci. 2009; 1155: 141-161.

8. Cuzick J , Sestak I, Forbes JF, et al. Anastrozole for prevention of breast cancer in high-risk postmenopausal women (IBIS-II): an international, double-blind, randomised placebo-controlled trial. Lancet. 2014; 383(9922): 1041-1048.

9. Maunsell E, Goss PE, Chlebowski RT, et al. Quality of life in MAP.3 (Mammary Prevention 3): a randomized, placebo-controlled trial evaluating exemestane for prevention of breast cancer. J Clin Oncol. 2014; 32(14): 1427-1436.

10. Weir HK, Thompson TD, Soman A, et al. The past, present, and future of cancer incidence in the United States: 1975 through 2020. Cancer. 2015; 121(11): 1827-1837.

11. Nguyen TL, Choi YH, Aung YK ,et al. Breast Cancer Risk Associations with Digital Mammographic Density by Pixel Brightness Threshold and Mammographic System. Radiology. 2018; 286(2): 433-442.

12. Shieh Y, Hu D, Ma L, et al. Breast cancer risk prediction using a clinical risk model and polygenic risk score. Breast Cancer Res Treat. 2016; 159(3): 513-525.

13. Allman R, Dite GS, Hopper JL, et al. SNPs and breast cancer risk prediction for African American and Hispanic women. Breast Cancer Res Treat. 2015; 154(3): 583-589.

14. Dite GS, MacInnis RJ, Bickerstaffe A, et al. Breast Cancer Risk Prediction Using Clinical Models and 77 Independent Risk-Associated SNPs for Women Aged Under 50 Years: Australian Breast Cancer Family Registry. Cancer Epidemiol Biomarkers Prev. 2016; 25(2): 359-365.

15. Brentnall AR, Cuzick J, Buist DSM, et al. Long-term Accuracy of Breast Cancer Risk Assessment Combining Classic Risk Factors and Breast Density. JAMA Oncol. 2018; e180174.

16. Michailidou K, Lindström S, Dennis J, et al. Association analysis identifies 65 new breast cancer risk loci. Nature. 2017; 551(7678): 92-94.

17. Easton DF, Pooley KA, Dunning AM, et al. Genome-wide association study identifies novel breast cancer susceptibility loci. Nature. 2007; 447(7148): 1087-1093.

18. Zhang B, Beeghly-Fadiel A, Long J, et al. Genetic variants associated with breast-cancer risk: comprehensive research synopsis, metaanalysis, and epidemiological evidence. Lancet Oncol. 2011; 12(5): 477-488.

19. Smith P, McGuffog L, Easton DF, et al. A genome wide linkage search for breast cancer susceptibility genes. Genes Chromosomes Cancer. 2006; 45(7): 646-655.

20. Pharoah PD, Antoniou A, Bobrow M, et al. Polygenic susceptibility to breast cancer and implications for prevention. Nat Genet. 2002; 31(1): 33-36.

21. Mavaddat N, Pharoah PD, Michailidou K, et al. Prediction of breast cancer risk based on profiling with common genetic variants. J Natl Cancer Inst. 2015; 107(5): 10.1093/jnci/djv036. Print 2015 May.

22. Rudolph A, Song M, Brook MN, et al. Joint associations of a polygenic risk score and environmental risk factors for breast cancer in the Breast Cancer Association Consortium. Int J Epidemiol. 2018; 47(2): 526-536.

23. Newman LA, Kaljee LM. Health Disparities and Triple-Negative Breast 
Cancer in African American Women: A Review. JAMA Surg. 2017; 152(5): 485-493.

24. DeSantis CE, Ma J, Goding Sauer A, et al. Breast cancer statistics, 2017, racial disparity in mortality by state. CA Cancer J Clin. 2017; 67(6): 439-448.

25. Coignet MV, Zirpoli GR, Roberts MR, et al. Genetic variations, reproductive aging, and breast cancer risk in African American and European American women: The Women's Circle of Health Study. PLoS One. 2017; 12(10): e0187205.

26. Huo D, Feng Y, Haddad S, et al. Genome-wide association studies in women of African ancestry identified 3q26.21 as a novel susceptibility locus for oestrogen receptor negative breast cancer. Hum Mol Genet. 2016; 25(21): 4835-4846.

27. Wang S, Qian F, Zheng Y, et al. Genetic variants demonstrating flipflop phenomenon and breast cancer risk prediction among women of African ancestry. Breast Cancer Res Treat. 2018; 168(3): 703-712.

28. Broeders M, Moss S, Nyström L, et al. The impact of mammographic screening on breast cancer mortality in Europe: a review of observational studies. J Med Screen. 2012; 19 Suppl 1: 14-25.

29. National Comprehensive Cancer Network. Breast Cancer Screening and Diagnosis V1.2017 Accessed, April, 6 2018];https://www.nccn. org/professionals/physician_gls/pdf/breast-screening.pdf.

30. Esserman LJ, WISDOM Study and Athena Investigators. The WISDOM Study: breaking the deadlock in the breast cancer screening debate. NPJ Breast Cancer. 2017; 3: 34-017-0035-5. eCollection 2017.

31. Layeequr Rahman R, Crawford S. Chemoprevention Indication Score: a user-friendly tool for prevention of breast cancer - pilot analysis. Breast. 2009; 18(5): 289-293.

32. Hum S, Wu M, Pruthi S, et al. Physician and Patient Barriers to Breast Cancer Preventive Therapy. Curr Breast Cancer Rep. 2016; 8(3): 158164.

33. Reimers LL, Sivasubramanian PS, Hershman D, et al. Breast Cancer Chemoprevention among High-risk Women and those with Ductal Carcinoma In Situ. Breast J. 2015; 21(4): 377-386.

34. Noonan S, Pasa A, Fontana V, et al. A Survey among Breast Cancer Specialists on the Low Uptake of Therapeutic Prevention with Tamoxifen or Raloxifene. Cancer Prev Res. (Phila). 2018; 11(1): 38-43.

35. Holmberg C, Bandos H, Fagerlin A, et al. NRG Oncology/National Surgical Adjuvant Breast and Bowel Project Decision-Making Project-1 Results: Decision Making in Breast Cancer Risk Reduction. Cancer Prev Res (Phila). 2017; 10(11): 625-634.

36. Evans DG, Howell SJ, Howell A. Personalized prevention in high risk individuals: Managing hormones and beyond. Breast. 2018.

37. Karavites LC, Allu S, Khan SA, et al. Awareness of preventive medication among women at high risk for breast cancer and their willingness to consider transdermal or oral tamoxifen: a focus group study. BMC Cancer. 2015; 15: 878-015-1893-6.

38. Freedman AN, Graubard BI, Rao SR, et al. Estimates of the number of US women who could benefit from tamoxifen for breast cancer chemoprevention. J Natl Cancer Inst. 2003; 95(7): 526-532.

39. Corbie-Smith G. The continuing legacy of the Tuskegee Syphilis Study: considerations for clinical investigation. Am J Med Sci. 1999; 317(1): 5-8.

40. Buseh AG, Stevens PE, Millon-Underwood S, et al. Community leaders' perspectives on engaging African Americans in biobanks and other human genetics initiatives. J Community Genet. 2013; 4(4): 483-494.

41. Durant RW, Wenzel JA, Scarinci IC, et al. Perspectives on barriers and facilitators to minority recruitment for clinical trials among cancer center leaders, investigators, research staff, and referring clinicians: enhancing minority participation in clinical trials (EMPaCT). Cancer. 2014; 120 Suppl 7: 1097-1105.

42. Fisher JA, Kalbaugh CA. Challenging assumptions about minority participation in US clinical research. Am J Public Health. 2011; 101(12): 2217-2222.

43. Durant RW, Legedza AT, Marcantonio ER, et al. Different types of distrust in clinical research among whites and African Americans. J Natl Med Assoc. 2011; 103(2): 123-130.

44. White-Means SI, Osmani AR. Racial and Ethnic Disparities in PatientProvider Communication With Breast Cancer Patients: Evidence From 2011 MEPS and Experiences With Cancer Supplement. Inquiry. 2017; 54: 46958017727104 .

45. Stickeler E, Fehm T, Schutz F, et al. Aromatase Inhibitors in the Prevention of Breast Cancer. Breast Care (Basel). 2015; 10(2): 141-142.

46. Vachon CM, Schaid DJ, Ingle JN,et al. A polygenic risk score for breast cancer in women receiving tamoxifen or raloxifene on NSABP P-1 and P-2. Breast Cancer Res Treat. 2015; 149(2): 517-523.

47. Cuzick J, Brentnall AR, Segal C, et al. Impact of a Panel of 88 Single Nucleotide Polymorphisms on the Risk of Breast Cancer in High-Risk Women: Results From Two Randomized Tamoxifen Prevention Trials. J Clin Oncol. 2017; 35(7): 743-750.

48. Popejoy AB, Fullerton SM. Genomics is failing on diversity. Nature. 2016; 538(7624): 161-164.

49. Feng Y, Rhie S, Huo D, et al. Characterizing Genetic Susceptibility to Breast Cancer in Women of African Ancestry. Cancer Epidemiol Biomarkers Prev. 2017; 26(7): 1016-1026.

50. Kincaid E. Largest genetic study of breast cancer in African Americans launched. Nat Med. 2016; 22(9): 966-966.

51. Banegas MP, John EM, Slattery ML, et al. Projecting Individualized Absolute Invasive Breast Cancer Risk in US Hispanic Women. J Natl Cancer Inst. 2016; 109(2): 10.1093/jnci/djw215. Print 2017 Feb. 